\title{
Liver steatosis is a component of the hepatocardiorenal syn- drome provoked by a high-lipid diet and activation of Ang II pathways in rats
}

\author{
Thuany Crisóstomo 1,2, Marco A. E. Pardal 3, Simone A. Herdy ${ }^{4}$, Humberto Muzi-Filho ${ }^{2,3}$, Debora B. Mello ${ }^{2}$, Rafael \\ Luzes ${ }^{2,3,4}$ and Adalberto Vieyra ${ }^{2,3,4, *}$ \\ 1 Leopoldo de Meis Institute of Medical Biochemistry, Federal University of Rio de Janeiro, Rio de Janeiro, \\ Brazil; thuany.crisostomo@bioqmed.ufrj.br (T.C.) \\ 2 National Center of Structural Biology and Bioimaging/CENABIO, Federal University of Rio de Janeiro, Rio \\ de Janeiro, Brazil; debmello@cenabio.ufrj.br (D.B.M.) \\ 3 Carlos Chagas Filho Institute of Biophysics, Federal University of Rio de Janeiro, Rio de Janeiro, Brazil; \\ marco.pardal@biof.ufrj.br (M.A.E.P.); humbertomuzi@biof.ufrj.br (H.M.-F.); rafaelluzes@biof.ufrj.br (R.L.) \\ 4 Graduate Program in Translational Biomedicine/BIOTRANS, University of Grande Rio, Duque de Caxias, \\ Brazil; herdysimone@biof.ufrj.br (S.A.H.) \\ * Correspondence: avieyra@biof.ufrj.br; Tel.: +55-21-3938-6520; Fax: +55-21-2280-8193
}

\begin{abstract}
Overweight/obesity is a growing pandemic nowadays that affects many organs and tissues. We have investigated whether a high-lipid diet provokes an imbalance between type 1 and type 2 angiotensin II (Ang II) receptors signaling, leading to liver alterations associated with previously described cardiovascular and kidney disturbances. Chronic administration of a high-lipid diet can provoke an hepatocardiorenal syndrome as the result of activation of the Ang II $\rightarrow$ type 1 receptor axis, which is completely counteracted by Ang-(3-4) the allosteric enhancer of the Ang $\mathrm{II} \rightarrow$ type 2 receptor pathway.
\end{abstract}

Keywords: hyperlipidic diet; overweight rats; non-alcoholic liver steatosis; arterial hypertension; increased renal sodium reabsorption; hepatocardiorenal syndrome

\section{Introduction}

We recently communicated that young rats chronically given a hypercaloric diet in which 70\% of calories come from lipids [1] (a "Western diet" [2]), developed overweight with increased visceral fat (perirenal and epididymal), hypertension, exacerbated active $\mathrm{Na}^{+}$reabsorption in kidney proximal tubule cells, and highly positive $\mathrm{Na}^{+}$balance [3]. We proposed that the cardiovascular and renal alterations resulted from hyperactivation of angiotensin II type 1 receptor $\left(A T_{1} R\right)$ signaling (the Ang $I I \rightarrow A T_{1} R$ axis of the renin-angiotensin-aldosterone system/RAAS), which was counteracted by administration of Ang-(3-4) (Val-Tyr), the shortest renin-angiotensin-derived peptide. Ang-(3-4) antagonizes several effects of Ang II in physiological and pathological conditions [4]. One of the mechanisms for the counteracting effect is the allosteric enhancing of Ang II binding to type 2 receptors by Ang-(3-4) [5], i.e., the activation of the Ang II $\rightarrow \mathrm{AT}_{2} \mathrm{R}$ axis of RAAS. Facing the concomitant cardiac and renal alterations, we can initially propose that the overweight resulting from chronic administration of a high-lipid (HL) diet can culminate with a secondary type $\mathrm{V}$ cardiorenal syndrome. In this type, a systemic pathology (such as for overweight/obesity) simultaneously affects the heart and the kidney [6].

Using ultrasound, we carried out further studies on the retroperitoneal and perirenal regions. The driving idea for this study came from the central role that the perirenal fat has in the activation of the Ang II $\rightarrow \mathrm{AT}_{1} \mathrm{R}$ axis of RAAS [7] and from the hypothesis that being overweight could provoke liver alterations. The stimulus of proinflammatory 
cytokines by the visceral fat contributes to insulin resistance and dyslipidemias, generalized inflammation and lipotoxicity, and the development of hepatic cirrhosis [8,9].

\section{Materials and Methods}

\subsection{Ethical Considerations}

The study was approved by the Committee for Ethical Use of Animals in Experimentation at the Federal University of Rio de Janeiro (protocol 075/19).

\subsection{Recording of Ultrasound Images}

Overweight and hypertensive rats that received the HL diet from 58 to 164 days of age and animals that were given a control chow (CTR) from the same manufacturer (PRAG Solutions, São Paulo, Brazil) during the same period [3] were anesthetized with isofluorane. Images were obtained with the high-resolution ultrasound Vevo® 2100 (FUJIFILM VisualSonics, Toronto, Canada) coupled to a $20-70 \mathrm{MHz}$ transducer by a single technologist at the National Center for Structural Biology and Bioimaging, as described by Marshall et al. [10] with slight modifications. Briefly, images were recorded from the right upper retroperitoneal region and the right liver lobe delimitated by the circle tool of the system. After transforming the images to the JPEG format, the hepatorenal index was calculated from the mean brightness and size in pixels from the two organs [11] using the software ImageJ (1.4.3.67 version).

\section{Results}

Figure 1 presents images of hepatorenal index rats given CTR or HL diets, obtained $48 \mathrm{~h}$ after administration of 4 doses by gavage, at $12 \mathrm{~h}$ intervals, of vehicle (water) or Ang-(3-4) (80 mg/kg) (Aminotech, Diadema, Brazil). Comparing the representative images shown in panels $\mathrm{A}$ and $\mathrm{B}$ demonstrate an accentuated increase of brightness in the liver (L) from the HL rat, without change in the brightness of kidney (K). The increase reached $100 \%$, as shown in the bargraph presented in panel $\mathrm{E}$, and reveals the presence of steatosis, which structural correlation is the percentual augment of fat deposits. Biochemically, it corresponds to ectopic deposits of triglycerides.
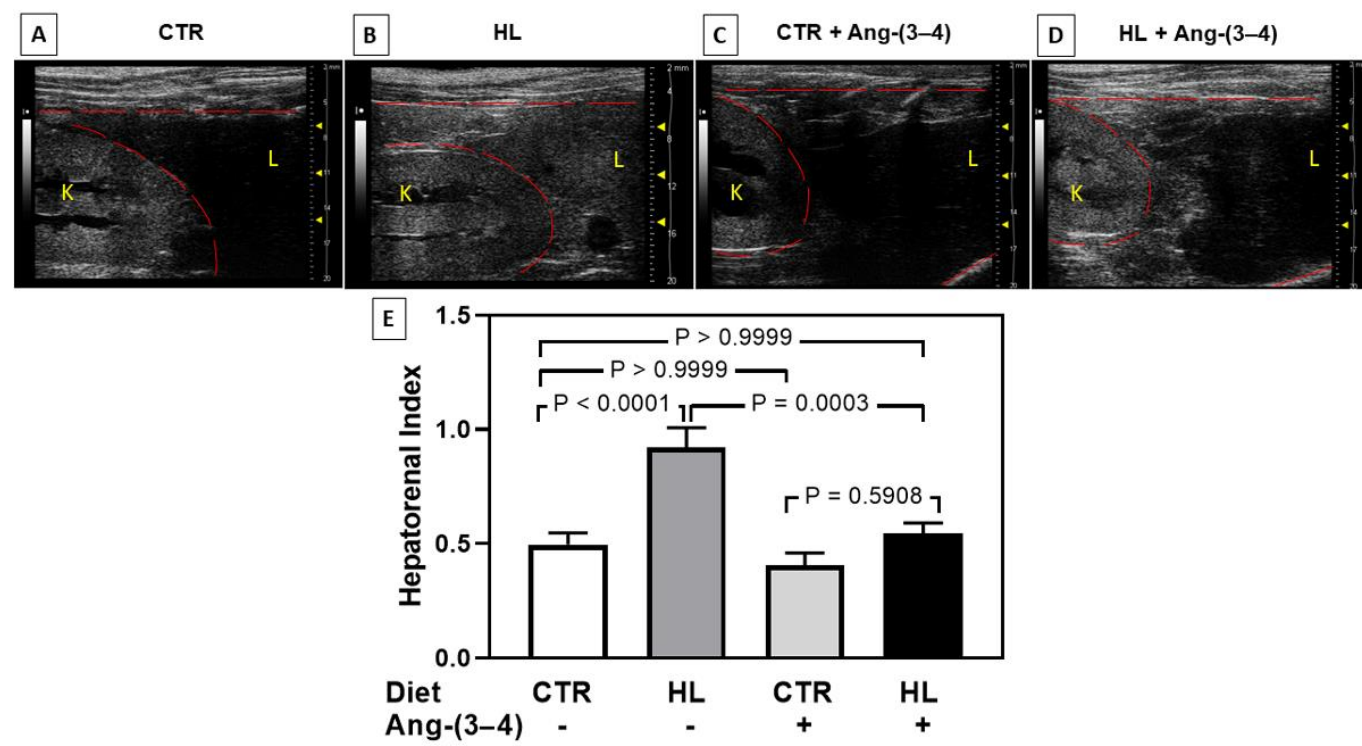

Figure 1. Hepatorenal index (mean hepatic brightness/mean renal brightness) in rats given control (CTR) and high-lipid content (HL) diets: effect of Ang-(3-4). (A, B, C, D) Representative abdominal ultrasound images from CTR, HL, CTR + Ang-(3-4), and HL + Ang-(3-4) rats, respectively. Ang-(34) was administered as described in the text. The organs are identified by their initial letters L (liver) and $\mathrm{K}$ (kidney), and the images were processed as described in the text. (E) Graphic representation of the pixels densities in CTR $(n=17)$, HL $(n=12)$, CTR + Ang-(3-4) $(n=12)$, and HL + 
Ang-(3-4) ( $n=14)$ rats. Differences were assessed by using one-way ANOVA followed by Bonferroni's test for selected pairs; $p$ values are indicated within the panel.

Figure 1 also confirms that the hepatic lesions provoked by the HL diet resulted from the activation of the Ang $\mathrm{II} \rightarrow \mathrm{AT}_{1} \mathrm{R}$ axis of RAAS, because they regressed rapidly (panels $\mathrm{C}, \mathrm{D}$, and $\mathrm{E}$ ) after stimulation of the Ang $\mathrm{II} \rightarrow \mathrm{AT}_{2} \mathrm{R}$ by its allosteric enhancer Ang-(3-4) [5]. The hepatic brightness returned to the levels found in CTR rats, which were not modified by Ang-(3-4), another evidence that Ang-(3-4) acts only in pro-hypertensive microenvironments [4].

\section{Discussion}

In a scenario of lipidic infiltration, such as that shown in Figure 1, the production and release of proinflammatory cytokines significantly increase [12]. The production of the hepatic Fetuin-A also increases and, therefore, the inhibition of the insulin cascade and the release of inflammatory cytokines [12]. At the same time, Fetuin-A allows saturated fatty acid to stimulate the type 4 Toll-like receptors (TLR4) of macrophages [13], thus accelerating the conversion of type M2 macrophages toward the M1 phenotype [14]. At the renal sinus fat level, Fetuin-A promotes the transition from a "protector" profile to a "lesional" one, propagating and amplifying the release of proinflammatory cytokines [14].

We previously demonstrated that activation of TLR4 is a central mechanism in the genesis of inflammatory cardiac lesions after acute renal injury [15], an example of type III cardiorenal syndrome [6]. Since Fetuin-A also alters the cardiac metabolism [14] and the overweight of rats receiving the HL diet is associated with hypertension, and molecular and RAAS-mediated alterations in renal $\mathrm{Na}^{+}$-transporting ATPases [3], the data from Figure 1 allows us to propose that the Western diet HL can lead to a hepatocardiorenal syndrome, an emerging concept in pathology [16].

The complete and faster regression of steatosis after administration of Ang-(3-4) also entails accentuated, rapid, and continuous lipolysis, probably resulting from the activation of both the adipose triglyceride lipase (ATGL) by cyclic AMP-dependent protein kinase (PKA) [17] and the hormone-sensitive lipase (HSL) by catecholamines [18], whose defects play a central role in obesity [19]. We conclude that, together with an activated cyclic AMP-dependent protein kinase coupled to the upregulated Ang II $\rightarrow \mathrm{AT}_{2} \mathrm{R}$ axis [5], these lipases antagonize the Ang $\mathrm{II} \rightarrow \mathrm{AT} 1 \mathrm{R} \rightarrow$ protein kinase $\mathrm{C}$ proinflammatory and antilipolytic signaling axis.

\section{Conclusions}

In summary, the observations communicated here provide valuable evidence regarding an hepatocardiorenal syndrome [16] induced by chronic administration of a diet with a high-lipid content, in which abnormal upregulation of the Ang-(3-4)-sensitive Ang II $\rightarrow \mathrm{AT}_{1} \mathrm{R}$ axis of RAAS culminates with connected liver steatosis (Figure 1), arterial hypertension [3], and augmented renal $\mathrm{Na}^{+}$reabsorption [3] as the prominent pathological disturbances.

Author Contributions: Conceptualization, T.C., S.A.H., H.M.-F., D.B.M., R.L. and A.V.; methodology, T.C., M.A.E.P., S.A.H. and D.B.M.; formal analysis, T.C., M.A.E.P., S.A.H. and D.B.M.; investigation, T.C., M.A.E.P., S.A.H. and D.B.M.; data curation, T.C., M.A.E.P., S.A.H. and D.B.M.; writing-original draft preparation, T.C., S.A.H., H.M.-F., R.L. and A.V.; writing - review and editing, T.C., S.A.H., H.M.-F., R.L. and A.V.; supervision, R.L. and A.V.; project administration, T.C., R.L. and A.V.; funding acquisition, T.C., H.M.-F., R.L. and A.V. All authors have read and agreed to the published version of the manuscript.

Funding: This research was funded by Brazilian Research Council/CNPq, grant numbers 307605/2015-9 and 440544/2018-1, Rio de Janeiro State Foundation FAPERJ, grant numbers E-26/202.963/2017, E-26/210.890/2019, E-26/201.909/2020 and E-26/200.866/2021, and the Brazilian 
Federal Agency for Support and Evaluation of Graduate Education/CAPES, grant numbers 88887.124150/2014-00, 88887.320213/2019-00 and 88887.623346/2021-00.

Institutional Review Board Statement: The study was conducted according to the guidelines of the Declaration of Helsinki, and approved by the Committee for Ethical Use of Animals in Experimentation at the Federal University of Rio de Janeiro (protocol code 075/19).

Data Availability Statement: Data available on request due to restrictions e.g., privacy or ethical.

The data presented in this study are available on request from the corresponding author.

Conflicts of Interest: The authors declare no conflict of interest. The funders had no role in the design of the study; in the collection, analyses, or interpretation of data; in the writing of the manuscript, or in the decision to publish the results.

\section{References}

1. Touati, S.; Meziri, F.; Devaux, S.; Berthelot, A.; Touyz, R.M.; Laurant P. Exercise reverses metabolic syndrome in high-fat diet-induced obese rats. Med. Sci. Sports Exerc. 2011, 43, 398-407.

2. Kopp, W. How western diet and lifestyle drive the pandemic of obesity and civilization diseases. Diabetes Metab. Syndr. Obes. 2019, 12, 2221-2236.

3. Luzes, R.; Crisóstomo, T.; Silva, P.A.; Iack, R.; de Abreu, V.G.; Francischetti, E.A.; A. Vieyra. Angiotensin-(3-4) normalizes blood pressure, decreases $\mathrm{Na}^{+}$and energy intake, but preserves urinary $\mathrm{Na}+$ excretion in overweight hypertensive rats. Biochim. Biophys. Acta Mol. Basis. Dis. 2021, 1867, 166012.

4. Dias, J.; Axelband, F.; Lara, L.S.; Muzi-Filho, H.; Vieyra, A. Is angiotensin-(3-4) (Val-Tyr), the shortest angiotensin II-derived peptide, opening new vistas on the renin-angiotensin system? J. Renin Angiotensin Aldosterone Syst. 2017, 18, 1470320316689338.

5. Axelband, F.; Dias, J.; Miranda, F.; Ferrão, F.M.; Reis, R.I.; Costa-Neto, C.M.; Lara, L.S.; Vieyra A. Angiotensin-(3-4) counteracts the Angiotensin II inhibitory action on renal $\mathrm{Ca}^{2+}$-ATPase through a cAMP/PKA pathway. Regul. Pept. 2012, 177, 27-34.

6. Ronco, C.; Ronco, F. Cardio-renal syndromes: a systematic approach for consensus definition and classification. Heart Fail. Rev. 2012, 17, 151-160.

7. Hall, J.E.; do Carmo, J.M.; da Silva, A.A.; Wang, Z.; Hall, M.E. Obesity-induced hypertension: interaction of neuro-humoral and renal mechanisms. Circ. Res. 2015, 116, 991-1006.

8. Garbarino, J.; Sturley, S. Saturated with fat: new perspectives on lipotoxicity. Curr. Opin. Clin. Nutr. Metab. Care. 2009, 12, 110116.

9. Loria, P.; Lonardo, A.; Anania, F. Liver and diabetes. A vicious circle. Hepatol. Res. 2013, 43, 51-64.

10. Marshall, R.H.; Eissa, M.; Bluth, E.I.; Gulotta, P.M.; Davis N.K. Hepatorenal index as an accurate, simple, and effective tool in screening for steatosis. Am. J. Roentgenol. 2012, 199, 997-1002.

11. Webb, M.; Yeshua, H.; Zelber-Sagi, S.; Santo, E.; Brazowski, E.; Halpern, Z.; Oren, R. Diagnostic value of a computerized hepatorenal index for sonographic quantification of liver steatosis. AJR Am. J. Roentgenol. 2009, 192, 909-914.

12. Larter, C.Z.; Chitturi, S.; Heydet, D.; Farrell, G.C. A fresh look at NASH pathogenesis. Part 1: the metabolic movers. J. Gastroenterol. Hepatol. 2010, 25, 672-690.

13. Pal, D.; Dasgupta, S.; Kundu, R.; Maitra, S.; Das, G.; Mukhopadhyay, S.; Ray, S.; Majumdar, S.S.; Bhattacharya, S. Fetuin-A acts as an endogenous ligand of TLR4 to promote lipid-induced insulin resistance. Nat. Med. 2012, 18, 1279-1285.

14. Trepanowski, J.F.; Mey, J.; Varady, K.A. Fetuin-A: a novel link between obesity and related complications. Int. J. Obes. (Lond). 2015, 39, 734-741.

15. Trentin-Sonoda, M.; da Silva, R.C.; Kmit, F.V.; Abrahão, M.V.; Monnerat Cahli, G.; Brasil, G.V.; Muzi-Filho, H.; Silva, P.A.; Tovar-Moll, F.F.; Vieyra, A. et al. Knockout of toll-like receptors 2 and 4 prevents renal ischemia-reperfusion-induced cardiac hypertrophy in mice. PLoS One. 2015, 10, e0139350.

16. Kazory, A.; Ronco, C. Hepatorenal syndrome or hepatocardiorenal syndrome: revisiting basic concepts in view of emerging data. Cardiorenal Med. 2019, 9, 1-7.

17. Pagnon, J.; Matzaris, M.; Stark, R.; Meex, R. C.; Macaulay, S.L.; Brown, W.; O'Brien, P.E.; Tiganis, T.; Watt, M.J. Identification and functional characterization of protein kinase A phosphorylation sites in the major lipolytic protein, adipose triglyceride lipase. Endocrinology, 2012, 153, 4278-4289.

18. Reynisdottir, S.; Wahrenberg, H.; Carlström, K.; Rössner, S.; Arner, P. Catecholamine resistance in fat cells of women with upper-body obesity due to decreased expression of $\beta 2$-adrenoceptors. Diabetologia. 1994, 37, $428-435$.

19. Langin, D.; Dicker, A.; Tavernier, G.; Hoffstedt, J.; Mairal, A.; Rydén, M.; Arner, E.; Sicard, A.; Jenkins, C.M.; Viguerie, N.; et al. Adipocyte lipases and defect of lipolysis in human obesity. Diabetes. 2005, 54, 3190-3197. 